\title{
MULTICRITERIA DYNAMIC SCHEDULING BY TOPSIS AND GoAl Programming
}

\author{
Amol Singh \\ IIM Rohtak, Rohtak Haryana, 124001, India
}

\begin{abstract}
The present research work presents an algorithm for dynamic scheduling problems that optimize several criteria simultaneously. This algorithm is based on dispatching rules, TOPSIS and goal programming and integrates them to solve the multi-criteria dynamic job shop scheduling problems. The selection of dispatching rules is made by identifying the worst performing criterion. A rule, which can improve system performance for the worst performing criterion is selected to dispatch the part under consideration. The proposed multi-criteria dynamic scheduling algorithm is evaluated and compared with best reported scheduling rules. The results obtained demonstrate that the suggested algorithm proves it superiority over individual dispatching rules under multi-criteria dynamic job shop environment.
\end{abstract}

Keyword: Dynamic scheduling; dispatching rules; Multi-criteria scheduling; Simulation; TOPSIS
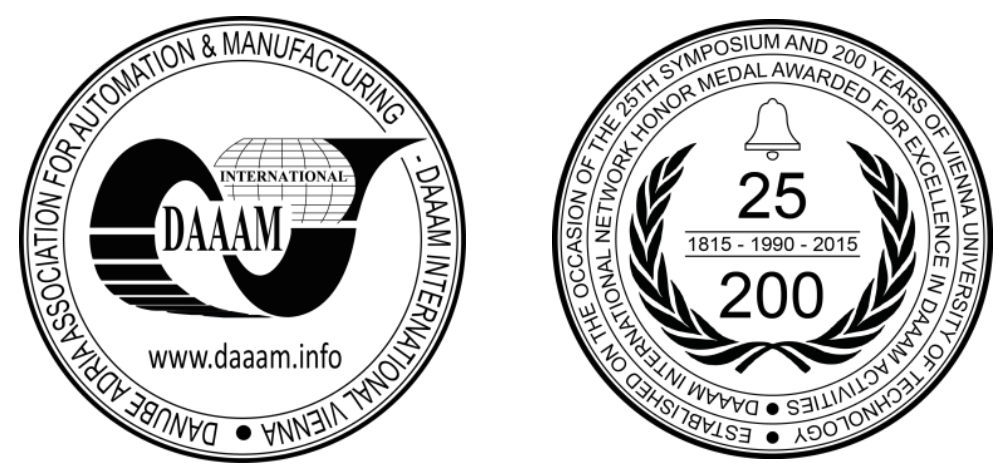

This Publication has to be referred as: Amol, A[mol] S[ingh] (2016). Multicriteria Dynamic Scheduling by Topsis and Goal Programming, Proceedings of the 26th DAAAM International Symposium, pp.0426-0434, B. Katalinic (Ed.), Published by DAAAM International, ISBN 978-3-902734-07-5, ISSN 1726-9679, Vienna, Austria DOI:10.2507/26th.daaam.proceedings.057 


\section{Introduction}

Scheduling is the most important activity of production planning and control. Scheduling literature ranges from static to dynamic scheduling problems, from single to multi-machine problems and from single to multi-criteria problems. The main objective of the scheduling problem is to optimize various performance measures, singly or jointly. A typical relationship between several types of scheduling problems has been summarized in Fig. 1. In manufacturing systems with large numbers of machines and several jobs with various routings competing for the various resources, the scheduling problem cannot be solved by the mathematical programming in a reasonable amount of time. In these instances, the use of efficient dispatching rules is the most dominant approach, which finds favour in the solution of the scheduling problems for its simplicity, ease of implementation and capability of providing quick solution. A solution that is optimal for a given criterion may not be optimal for some other criterion. In many practical situations, it would be thus desirable to achieve a solution that is best with respect to a number of different criteria simultaneously.

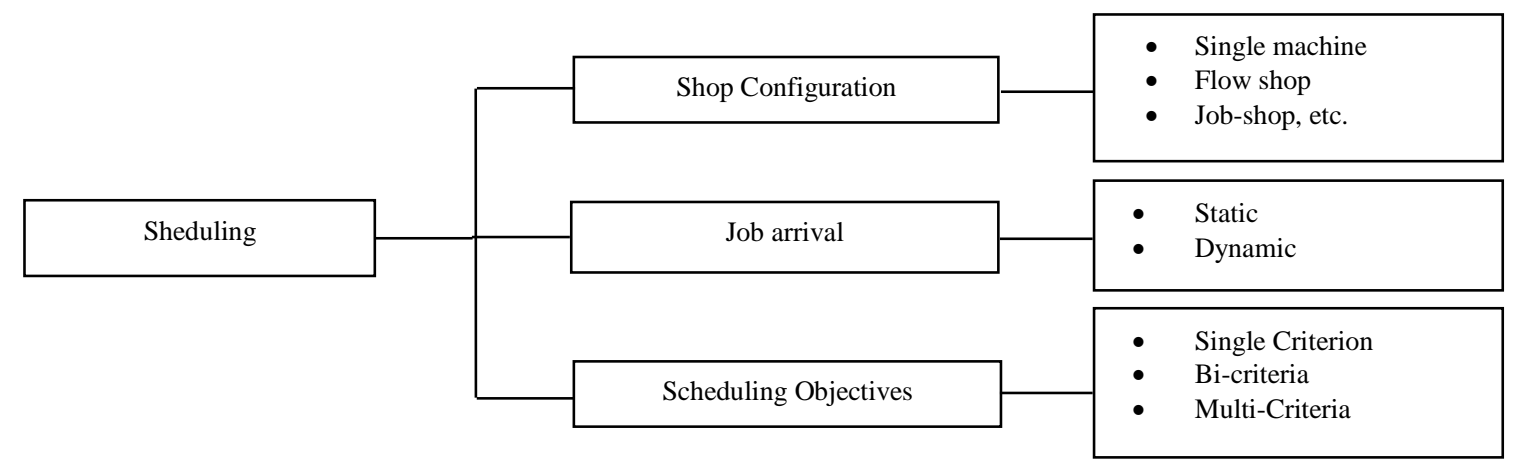

Fig. 1. A typical scheduling problem relationships

The research on bi-criteria and multi-criteria scheduling can be categorized under static and dynamic scheduling as shown in Fig. 1. Further, under static and dynamic category it can be categorized in four different types of models, viz. single machine bi-criteria \& multiple criteria scheduling, multiple machine bi-criteria \& multiple criteria scheduling. Most of the research done so far is on static scheduling problems and especially on single machine / flow shop bi-criteria static scheduling problems. Single machine bi-criteria scheduling problems were addressed by several researchers [1, 2]. Most of the work on multiple machines bi-criteria scheduling has been addressed in the literature under flow shop configuration [3,4,5,6,7]. Multi-criteria flow shop scheduling problems were also addressed by several researchers $[8,9,10]$. Yenisey [11] presented the review of multi-objective flow shop scheduling problems and clearly identified a need to address the multi-objective scheduling problems. Loukil et al. [10] reported a review of research on multi-criteria and bi-criteria scheduling approaches and suggested that Interactive approach and Goal programming are more suitable in real case studies.

Schnits [12] studied the multi-criteria dynamic scheduling problem for FMS. Gao [13] reported harmony search algorithm to solve the multi-objective flexible job shop scheduling problem. Most of the research on multiobjective flexible job shop scheduling has been reported under static environment [14, 15]. Fattahi [16] developed a mathematical model for dynamic flexible job shop scheduling problem which minimizes a weighted sum of make-span and stability. Qiu [17] reported a hybrid approach based on artificial intelligence and priority dispatching rules to solve the dynamic online job shop scheduling problem. Yao [18] proposed a multi-objective dynamic scheduling approach that combines three attributes based on a hybrid multiple attribute decision making technique. Nguygen [19] developed four new multi-objective genetic programming-based hyperheuristic methods for automatic design of scheduling policies, including dispatching rules and due-date assignment rules for job shop environments. Shen [20] applied evolutionary algorithms to solve the multi-objective dynamic flexible job shop scheduling problems. Job shop scheduling with multi-objective has been extensively investigated; however, multi-objective stochastic job shop scheduling problem is rarely considered [21]. Hoogeveen [21] reported the literature review on multi-criteria scheduling and highlighted a need of research on multi-criteria dynamic job shop scheduling. Job shop scheduling models have extensive practical applications. The lack of research in this domain has been due to the complex nature of scheduling problems. Hence, in the present research work, a hybrid algorithm based on TOPSIS and goal programming is developed to solve the multi-criteria dynamic job shop scheduling problem under machine breakdown conditions.

The reminder of the paper is organized as follows. Section 2 describes the multi-criteria job shop scheduling problem along with the considered input parameters for the study. Section 3 presents the multi-criteria job shop scheduling algorithm. Section 4 describes the simulation model to solve the MCDJSS problem. Section 5 reports the results of the simulation experiments. Section 6 concludes the study and indicates the scope for future research. 


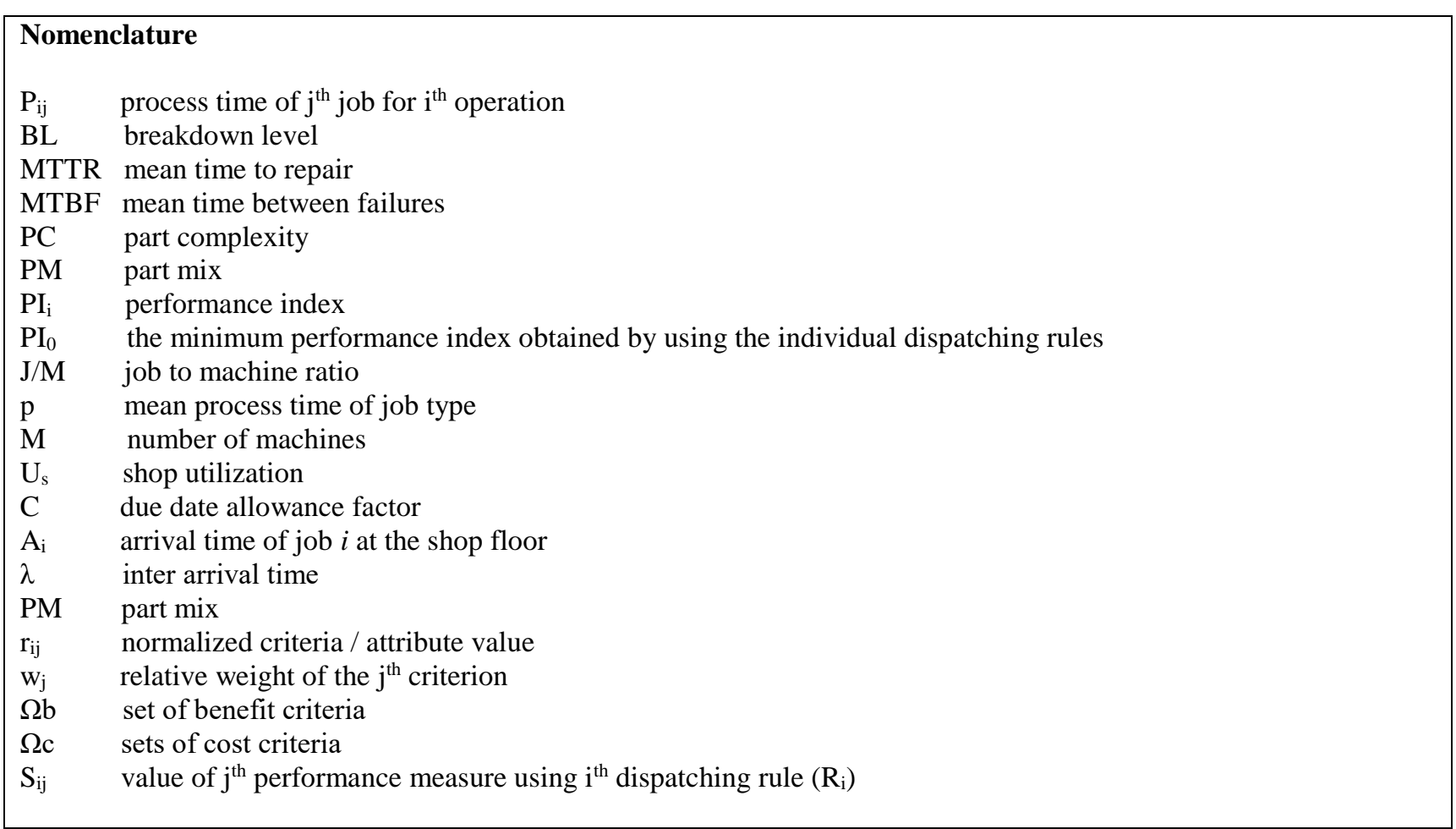

\section{Problem Statement}

The scheduling problem considered in the present study can be defined as follows: jobs arrive continuously into the system to be processed on multiple machines; the processing time of the job is pij. The task is to find the processing sequence of the jobs waiting machining at a required machine while minimizing several performance measures simultaneously. In order to find the processing sequence, dispatching rules are applied and the performance of the system is monitored continuously. Swapping of dispatching rules is allowed only when the performance index deviates from the pre-defined limit.

The considered manufacturing system is defined by two sets of parameters: system operating parameters and system configuration parameters. The system operating parameters are breakdown level (BL), mean time to repair (MTTR), utilization level and due dates while the system configuration parameters are number of job types to number of machine types $(\mathrm{J} / \mathrm{M})$ ratio, part complexity $(\mathrm{PC})$ and part mix $(\mathrm{PM})$. These parameters along with their levels are discussed below:

\subsection{Break down level}

Machine breakdown level (BL) is defined by the ratio of MTTR over the sum of MTTR and MTBF. In the simulation study, five values of breakdown levels i.e. $0 \%, 2.5 \%, 5 \%, 7.5 \%$ and $10 \%$ have been considered.

$$
B L=\frac{M T T R}{M T T R+M T B F}
$$

\subsection{Mean time to repair}

This is the average time to repair a machine and bring it back to acceptable operating condition. It includes the actual time spent on arranging spares and resources and then restoring the machine to make it operation worthy. Five levels of mean time to repair i. e. p, 2.5p, 5p, 7.5p and 10p have been considered.

\subsection{Arriva pattern, utilization and due date}

In the present study, the inter-arrival times for jobs are generated from the exponential distribution with mean calculated from the equation given below [22]:

$$
\lambda=\frac{p}{M \times U_{s}}
$$


In the tardiness based analysis, the due date of an arriving job is found from the following total work content relation [22].

$$
D_{i}=A_{i}+\left(C+\sum_{k=1}^{k=n i} p_{i k}\right)
$$

\subsection{Part complexity}

Part complexity (PC) is defined as the number of operations needed to complete the processing of a job [23]. In the present study, part complexity is defined by the average number of operations needed to complete the processing of a job and four; five and six operations have been selected for three levels of complexity. A job requiring more operations to complete its processing is said to be more complex than a job that requires fewer operations.

\subsection{Shop configuration}

In the present simulation study, ten machines with eight, ten and sixteen job types have been selected for study with three levels of shop configurations. In the first configuration, number of job types is less than the number of machine types $\mathrm{J}<\mathrm{M}$ (i. e. 8 job types with 10 machine types). In the second configuration, number of job types is equal to the number of machine types $\mathrm{J}=\mathrm{M}$ (i.e. 10 job types with 10 machine types) and in the third configuration, number of the job types is more than number of machine types $\mathbf{J}>\mathrm{M}$ (i.e. 16 job types with 10 machine types).

\subsection{Part mix}

Three levels of part mix (PM) have been considered for evaluating the performance of multi-criterion dynamic scheduling methodology. Part mix is based on the number of processing operations required in a part type. In the first part mix, it is assumed that all part types have equal volume of production. The second and third part mix have been generated by considering the industrial data given by Muhlemann [24]. From these data a cumulative probability distribution has been generated for obtaining the second and third part mix.

The processing times and processing sequence data for job types with the above discussed shop configuration parameters are generated randomly and used for the further analysis.

\section{Multi-criteria dynamic scheduling methodology}

In most production scheduling scenarios, the consideration of one criterion is not adequate to satisfy customer's due dates and manufacturing plan simultaneously. A production schedule is usually evaluated with respect to several criteria considering, for instance, overall production time (make-span), average job flow time, average job tardiness, number of tardy jobs. It is often desirable to achieve a solution that is best with respect to a number of different criteria simultaneously. Such multi-criteria problems can be solved by using the goal programming approach. In this method, all the objectives are assigned target levels for achievement and these target levels are treated as goals. An attempt is then made to find an optimal solution that comes as close as possible to the targets in order of the specified priorities by optimizing one objective at a time. The objective function using goal programming can be described as given below:

Minimize $\left(\mathrm{F}_{\mathrm{i}}\right)$ such that $\left(\mathrm{OF}_{1}, \mathrm{OF}_{2}, \mathrm{OF}_{3}, \ldots \ldots . . . \mathrm{OF}_{\mathrm{M}}\right)$ are $\max / \min$

In this formulation, OF1, OF2, OF3.....OFM are the decision variables (goals). Max / Min depends on the user specification that defines the goal by assuming the benefit criterion (larger the best) or cost criterion (smaller the best). During optimization process, the decision variable that is the farthest from its predefined target value is given the highest priority. Thus, this method solves one goal problem at a time with the highest priority goal. This concept of goal programming has been used for developing a hybrid algorithm to solve the multiple criteria dynamic scheduling problem.

A dynamic scheduling problem by considering $\mathrm{N}$ number of performance measures with a set of $\mathrm{M}$ alternative dispatching rules is solved by integrating the TOPSIS and goal programming approaches. The steps of the proposed hybrid algorithm that integrates the TOPSIS and goal programming approaches is explained below:

\subsection{Technique to similarity to ideal solution (TOPSIS)}

TOPSIS is a popular approach to solve the multi-criteria decision making (MCDM) problems. The ideal solution or positive ideal solution is a solution which maximizes the benefit criteria and minimizes the cost criteria, whereas the negative ideal solution maximizes the cost criteria and minimizes the benefit criteria. In general, the main aim of positive ideal and negative ideal solution is to maximize the benefit criteria and minimize the cost criteria. This 
method works on the principle that the chosen alternative has shortest distance from ideal solution and longest distance from negative ideal solution.

Suppose a multi-criteria dynamic scheduling problem is solved by using the $\mathrm{m}$ number of dispatching rules, $\mathrm{DR}_{1}, \mathrm{DR}_{2}, . . \mathrm{DR} \mathrm{R}_{\mathrm{m}}$, and $\mathrm{n}$ criteria $\mathrm{C}_{1}, \mathrm{C}_{2}, . . \mathrm{C}_{\mathrm{n}}$. Each dispatching rules is evaluated with respect to $\mathrm{n}$ considered criteria. All the performance rating/value obtained by each dispatching rule with respect to each criterion forms a decision matrix dennoted by $\mathrm{X}=\left(\mathrm{x}_{\mathrm{ij}}\right)_{\mathrm{m} \times \mathrm{n}}$. Suppose $\mathrm{W}=\left(\mathrm{w}_{1}, \mathrm{w}_{2}, . . \mathrm{w}_{\mathrm{n}}\right)$ are the relative weights assigned to $\mathrm{n}$ considered criteria, where $\sum_{j=1}^{n} w_{j}=1$. The multi-criteria rating of each rule is decided by using the TOPSIS as explained below:

Step 1: The values of the decision matrix $X$ are in different units hence, these values are converted into nondimensional ratio for comparison by using the normalization process. The normalize decision matrix $\mathrm{X}=\left(\mathrm{x}_{\mathrm{ij}}\right)_{\mathrm{m} \times \mathrm{n}}$ is computed by using the following equation.

$$
r_{i j}=\frac{x_{i j}}{\sqrt{\sum_{i=1}^{n} x_{i j}^{2}}} \mathrm{i}=1,2 \ldots \mathrm{m} ; \mathrm{j}=1,2, \ldots . \mathrm{n}
$$

Step 2: Calculate the weighted normalized decision matrix $V=\left(\mathrm{v}_{\mathrm{ij}}\right)_{\mathrm{m} \times \mathrm{n}}$ by using the following relation:

$$
v_{i j}=w_{j} \times r_{i j} \quad \mathrm{i}=1,2 \ldots \mathrm{m} ; \mathrm{j}=1,2, \ldots . . \mathrm{n}
$$

Step 3: Determine the ideal and negative ideal solutions by using the following equations.

$$
\begin{aligned}
& A^{*}=v_{1}^{*}, v_{2}^{*} \ldots \ldots \ldots . . v_{n}^{*}\{(\operatorname{maxj} v i j \mid \mathrm{j} \in \Omega \mathrm{b}),(\operatorname{minj} \mathrm{vij} \mid \mathrm{j} \in \Omega \mathrm{c})\} \\
& A^{-}=v_{1}^{-}, v_{2}^{-} \ldots \ldots \ldots . . v_{n}^{-}\{(\operatorname{minj} v i j \mid \mathrm{j} \in \Omega \mathrm{b}),(\operatorname{maxj} v i j \mid \mathrm{j} \in \Omega \mathrm{c})\}
\end{aligned}
$$

Step 4: Compute the separation measures (Euclidean distances) of each dispatching rule from the positive ideal solution and negative ideal solution by using the following relations.

$$
\begin{aligned}
& D_{i}^{*}=\sqrt{\sum_{j=1}^{n}\left(v_{i j}-v_{j}^{*}\right)}, \mathrm{i}=1,2 \ldots \mathrm{m} \\
& D_{i}^{-}=\sqrt{\sum_{j=1}^{n}\left(v_{i j}-v_{j}^{-}\right)}, \mathrm{i}=1,2 \ldots \mathrm{m}
\end{aligned}
$$

Step 5: Calculate the relative closeness of each dispatching rule by using each pair of separation measure as computed in step 4. The relative closeness of dispatching rule $\mathrm{DR}_{\mathrm{i}}$ with respect to $\mathrm{A} *$ is calculated as follow:

$$
R C_{i}=\frac{D_{i}^{-}}{D_{i}^{*}+D_{i}^{-}}
$$

Step 6: Determine the preference order by arranging the dispatching rules in the descending order of their relative closeness as computed in step 5. The best multi-criteria dispatching rule $\left(\mathrm{DR}_{\mathrm{i}}\right)$ is one with the highest relative closeness $\left(\mathrm{RC}_{\mathrm{i}}\right)$ to the ideal solution.

\subsection{Multi-criteria dynamic scheduling algorithm}

Step 1. Dynamic scheduling problem is solved by using each considered dispatching rules. The value of each performance measure is computed by using all considered rules one by one. In case of cost/benefit criteria the minimum/maximum value of each performance measure from among the calculated ones is determined and the corresponding dispatching rule for the performance measure under consideration is identified. If $S_{0 j}$ is the minimum/maximum value of $\mathrm{j}^{\text {th }}$ performance measure, then,

$$
S_{0 j}=\min / \max \left(s_{i j}\right) \forall \mathrm{i}=1 \text { to } \mathrm{M} \text { and } \mathrm{j}=1 \text { to } \mathrm{N}
$$

The rules corresponding to $S_{01}, S_{02} \ldots \ldots$ and $S_{0 N}$ have been identified as $R_{01}, R_{02} \ldots \ldots$ and $R_{0 N}$. 
Step 2. Once all the input parameter are set, the simulation is started by implementing the dispatching rule DR that is identified by applying the steps of TOPSIS algorithm explained in subsection 4.1. During the simulation, rule DR is used to prioritize the job in the queue of each machine.

Step 3: The performance of the system is monitored continuously. As simulation progresses, the actual value of the performance measures will deviate from the minimum value as determined in step 1, a deviation factor is computed for each of the performance measures to determine the percentage deviation in its value i. e.

$$
D F_{j}=\frac{S_{j} \text { current }-S_{0 j}}{S_{0 j}} \times 100 \forall \mathrm{j} \varepsilon(1 \text { to } \mathrm{N})
$$

Step 4. Deviation factor indicates the percentage deviation in the value of performance measure with respect to its minimum value. It may be noted that higher the deviation factor, worse is the corresponding system performance. The deviation factor for each performance measure is continuously monitored and the difference between the lowest deviation factor and highest deviation factor of the considered performance measures is determined with the occurrence of each event (i. e. arrival or departure of a job into the system) by the relation

$$
\left|D F_{\max }-D F_{\min }\right| \geq \% \text { ap }
$$

Step 5. If the difference in the deviation factors is more than the allowable percentage (ap) (i.e. specified by the user) then the existing dispatching rule is swapped by a new rule. Deviation factors as identified in step 3 are arranged in descending order and the performance measure corresponding to the highest deviation factor is considered for improvement. The rule that can improve the performance corresponding to the highest deviation factor is chosen to replace the current dispatching rule from $\left(\mathrm{R}_{01}, \mathrm{R}_{02} \ldots . . \mathrm{R}_{0 \mathrm{~N}}\right)$.

$\mathbf{S}$

tep 6. At the end of simulation run the values of all the taken performance measures are collected and finally, the system performance index is calculated by the relationship given below and compared with the performance index of rule $\mathrm{DR}_{\mathrm{i}}$.

$$
P I_{j}=\sum \frac{S_{j}}{S_{0 j}} \forall \mathrm{j} \varepsilon(1 \text { to } \mathrm{N})
$$

\section{Simulation model}

The present simulation study considers three shop configurations with varying number of job types to number of machine types ratio (i. e. $\mathrm{J} / \mathrm{M}>1, \mathrm{~J} / \mathrm{M}=1, \mathrm{~J} / \mathrm{M}<1$ ) for the evaluation of proposed methodology. The number of operations for each job type is assumed to be uniformly distributed in the range of (1-9) with uniformly distributed processing times in the range of (1-9). Job arrival in the system is assumed to follow exponential distribution with mean inter-arrival time between parts based on shop utilization. As the performance of multi criteria methodology is evaluated in the presence of machine breakdowns, down time of individual machines has been incorporated irrespective of whether it occurs due to tool breakage, tool adjustment, machine breakdown etc. Mean time between failure (MTBF) and mean time to repair (MTTR) are assumed to follow Gamma distribution. Busy time approach has been chosen for generating the breakdown times (i. e. a machine can fail only while performing an operation on a job). Law and Kelton [25] suggested that in the absence of real time data busy time distribution is most likely to be a Gamma distribution with a shape parameter of 0.7. They also suggested that Gamma distribution with a shape parameter of 1.4 is appropriate for generating the repair time. Thus the busy time between two successive failures is assumed to follow a Gamma distribution with $\alpha=0.7$ and $\beta=\operatorname{MTTR} \times(\mathrm{e} / 1-\mathrm{e}) \times 0.7$ and the duration of each breakdown is also assumed to follow a Gamma distribution with $\alpha=1.4$ and $\beta=$ MTTR/1.4. Where,

$$
e=\frac{M T B F}{M T B F+M T T R}
$$

Simulation models were developed in PROMODEL simulation software. Each simulation model has been run for 2000 completed jobs after attaining a steady state. The following general assumptions were made during the undertaken simulation studies. (i) Machines are not continuously available for processing and there are interruptions owing to breakdowns. If a machine breaks down, no operation can be performed until the end of the time of repair. (ii) When a machine breaks down, its repair starts immediately. (iii) Repaired machine works as efficiently as new machine. (iv) A machine can fail only while performing an operation on a job. 
The improvement in the system performance by using the proposed multi-criteria methodology is calculated by using the following relationship.

Percentage improvement $=\frac{P I_{i}-P I_{0}}{P I_{0} \times 100}$

Two objective functions are formulated by considering flow time based and tardiness based performance measures. The first objective function $\left(\mathrm{OF}_{1}\right)$ focuses on the flow time based performance measures and minimizes the mean, maximum, and variance of flow time simultaneously. The second objective function $\left(\mathrm{OF}_{2}\right)$ focuses on the tardiness based performance measures and minimizes mean \& maximum tardiness and number of tardy jobs simultaneously. The performance of multi criteria dynamic scheduling methodology is evaluated in varying system operating parameters with constant system configuration parameters (VOP-CSCP) environment and varying system configuration parameters with constant system operating parameters (VSCP-COP) by using $7 \& 6$ dispatching rules for flow time based \& tardiness based performance measures respectively. Dispatching rules FIFO, SPT, AT, AT-RPT, FDD, PT+PW, and PT+PW+FDD are considered for flow time based performance measures and the rules FIFO, SPT, $\mathrm{EDD}, \mathrm{ODD}, \mathrm{PT}+\mathrm{PW}, \mathrm{PT}+\mathrm{PW}+\mathrm{ODD}$ are considered for tardiness based performance measures. The brief description of all the above considered dispatching rules is given in the research papers [26, 27, 28]. In (VOP-CSCP) environment, by following a full factorial design approach, $200(=5 \times 5 \times 8) \& 175(=5 \times 5 \times 7)$ simulations runs were carried out for $\mathrm{OF}_{1} \&$ OF2 respectively. Similarly, in (VSCP-COP) environment, $351(=3 \times 3 \times 3 \times 13) \& 324(=3 \times 3 \times 3 \times 12)$ simulations runs were carried out for $\mathrm{OF}_{1} \& \mathrm{OF}_{2}$ respectively. Each experiment was repeated for each value of allowable percentage (ap) that was increased from 1 to $20 \%$ in steps of $1 \%$.

\section{Results and discussion}

The results are presented in terms of percentage improvement obtained by applying the proposed multi criteria dynamic scheduling methodology in comparison with the best performing individual dispatching rule. The analysis of the results based on flow time based objective function indicates that the, performance improvements are $20 \%$ \& $9 \%$ at $0 \% \& 10 \%$ breakdown levels respectively, when the repair time is $2.5 \mathrm{p}$ as shown in Fig. 2 . This trend indicates that as the breakdown level increases improvement in the performance decreases. This trend is also verified by the results obtained by using the tardiness based objective function under same operating conditions as shown in Fig. 2. During analysis, higher percentage improvement has been observed when there are few breakdowns of long duration compared with the case when there are relatively more breakdowns of short duration under identical breakdown level as shown in Fig. 3. However, in some cases performance improvement decreases with an increase in MTTR. During analysis, more improvement has been observed in flow time based objective function in comparison to tardiness based objective function

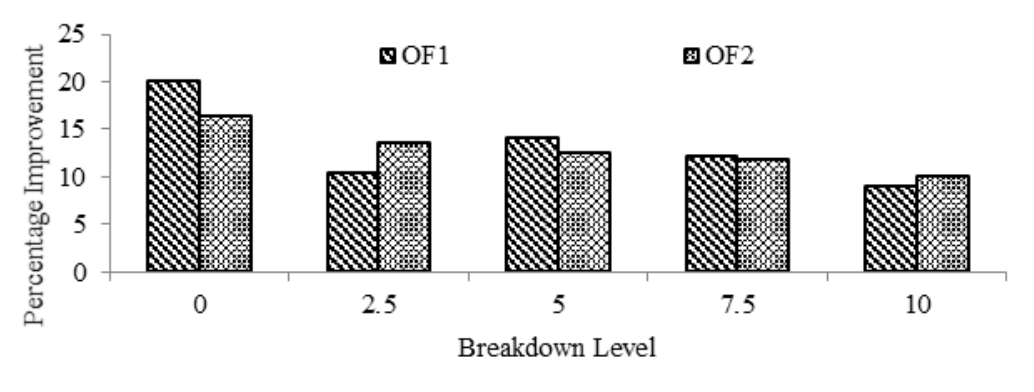

Fig. 2. Percentage of performance improved for OF1 \& OF2 at different breakdown levels.

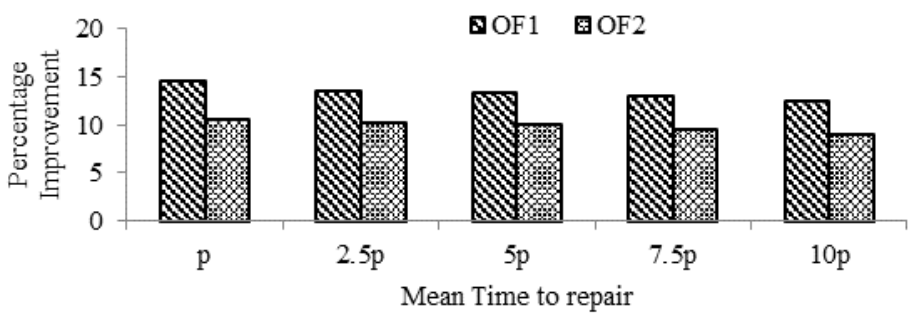

Fig. 3. \% of performance improvement for OF1 \& OF2 at different MTTR levels 
The results of flow time based \& tardiness based objectives are presented in terms of performance improvement at several levels of part complexities and $\mathrm{J} / \mathrm{M}$ ratio as shown in Fig. 4. Performance improvement of $13.0 \%$ and $9.8 \%$ has been observed when $\mathrm{J} / \mathrm{M}>1$ and $\mathrm{J} / \mathrm{M}<1$ respectively. This trend indicates that an increase in $\mathrm{J} / \mathrm{M}$ ratio increases the percentage improvement in the performance. In this case, $13.5 \%$ maximum improvement has been observed during analysis as shown in Fig. 4. At $\mathrm{PC}=4$ and $\mathrm{PC}=6$, the percentage improvement in performance index is 8.67 and 13.67. This trend indicates that the proposed methodology works more efficiently in a system that has high part complexity in comparison to low part complexity. The results of tardiness based performance measures indicate that the performance improvement at higher values of part complexities is more in comparison to lower values of part complexities. The performance improvement lies in the range of $11.60 \%$ to $16.85 \%$. It has been again verified by the trend that the improvement is more when $\mathrm{J} / \mathrm{M}>1$ than when $\mathrm{J} / \mathrm{M}<1$. The performance of multi-criteria methodology depends on the selection of efficient dispatching rules. This is because multi criteria dynamic scheduling algorithm continuously monitors the attained values of performance measures and whenever, the performance measures deteriorate beyond a pre-specified threshold, the swapping of dispatching rules is allowed and best rule corresponding to the worst performance measure is implemented. In all the considered cases, the proposed methodology improves the performance of the system.

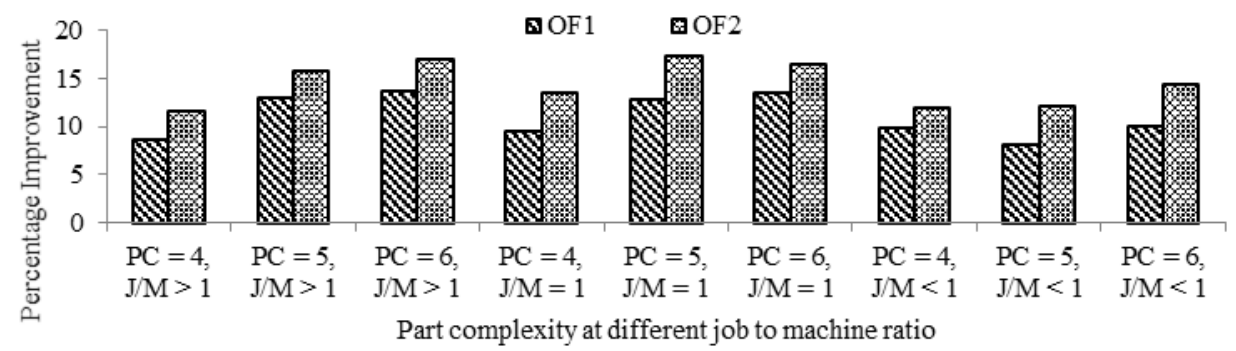

Fig. 4. $\%$ of performance improved for OF1 \& OF2 with different part complexity and job to machine ratio at BLs = $5 \%$, MTTR $=5 \mathrm{p}$

\section{Conclusions}

In the present study multi-criteria methodology based on goal programming and TOPSIS has been applied to solve the dynamic job shop scheduling problems under the random machine breakdowns. The multi-criteria methodology minimizes flow time and tardiness based performance measures simultaneously. The performance of the multi criteria methodology has been studied by considering several values of number of job types to number of machine type's ratio, breakdown parameters, part complexity and part mix. It has been demonstrated that the system performance improves by using the proposed multi-criteria dynamic scheduling algorithm in comparison with best existing dispatching rules. It has also been observed that the proposed methodology works more efficiently in a system that has job to machine ratio more than one and higher part complexity in comparison with a system having job to machine ratio less than one and lower part complexity. Further, it can be concluded that multi-criteria dynamic scheduling methodology based on TOPSIS and Goal programming improves the performance of dynamic manufacturing system in comparison with best existing dispatching rules.

The study on multi-criteria dynamic scheduling presented in this research paper could be extended in various ways. Firstly, more case studies on manufacturing systems engaged in diversified operations could underline the practical usefulness of the hybrid methodology as derived from the experimental results. Secondly, future research could consider the preventative maintenance type breakdowns, rush order etc. in the system. Finally, research can be extended by developing more hybrid approaches for multi-criteria dynamic scheduling problem under random or preventative maintenance type breakdowns.

\section{References}

[1] Su, L. H., Chou, F. D., Heuristic for scheduling in a bi-criteria single machine problem, Journal of Chinese Institute of Industrial Engineers, 18, (2001), 39-46.

[2] Koksalan, M., Keha, A. B., Using genetic algorithms for single-machine bicriteria scheduling problems, European Journal of Operational Research, 145, (2003), 543-556.

[3] Lio, C. J., Yu, W. C., Joe, C. B., Bicriterion scheduling in the two-machine flowshop, Journal of the operational research society, 48, (1997), 929-935.

[4] Li, Y., Li, X., Gupta, J.N.D., Solving the multi-objective flowline manufacturing cell scheduling problem by hybrid harmony search, Expert Systems with Applications, 42, (2015), 1409-1417.

[5] Allahverdi, A., Aldowaisan, T., No-wait flow shop with bi-criteria of make span and total completion time, Journal of the operational research society, 53, (2002), 1004-1015. 
[6] Gupta, JND., Ho, Jc., Webster, S., Bi criteria optimization of the make span and mean flow time on two identical parallel machines, Journal of Operational Research Society, 51, (2000), 1330-1339.

[7] Ho, J. C., Wong, J. S., Makespan minimization for parallel identical processors, Naval Research Logistics, 42 , (1979), 935-948.

[8] Teghem, J., Tuyttens, D., Ulungu, E. L., An interactive heuristic method for multi objective combinatorial optimization, Computer and Operations research, 27, (2000), 621-634.

[9] Allouche, M. A., Aouni, B., Martel, J-M, Loukil, T., Solving multi-criteria scheduling flow shop problem through compromise programming and satisfaction functions, European Journal of Operational Research, 192, (2009), 460-467.

[10] Loukil, T., Teghem, J., Tuyttens, D., Solving multi-objective production scheduling problems using metaheuristics, European Journal of Operational Research, 161, (2005), 42-61.

[11] Yenisey, M. M., Yagmahan, B., Multi-objective permutation flow shop scheduling problem: Literature review, classification and current trends, Omega, 45, (2014), 119-135.

[12] Schnits, B., Multi-criteria optimisation-based dynamic scheduling for controlling FMS, International Journal of Production Research, 50, (2012), 6111-6121.

[13] Gao, K.Z., Suganthan, P.N., Pan, Q.K., Chua, T.J., Cai, T.X., Chong, C.S., Pareto-based grouping discrete harmony search algorithm for multi-objective flexible job shop scheduling, Information Sciences, 289, (2014), 7690.

[14] Fattahi, P., Mehrabad, M. S., Jolai, F., Mathematical modelling and heuristic approaches to flexible job shop scheduling problems, Journal of Intelligent Manufacturing, 18, (2007), 331-342.

[15] Ozguven, C., Ozbakir, L., Yavuz, Y., Mathematical models for job-shop scheduling problems with routing and process plan flexibility, Applied Mathematical. Modelling, 34, (2010), 1539-1548.

[16] Fattahi, P., Fallahi, A., Dynamic scheduling in flexible job shop systems by considering simultaneously efficiency and stability, CIRP Journal of Manufacturing Science Technology, 2, (2010), 114-123.

[17] Qiu, X., Lau, H.Y.K., An AIS-based hybrid algorithm with PDRs for multi-objective dynamic online job shop scheduling problem, Applied Soft Computing 13, (2013), 1340-1351.

[18] Yao, S., Jiang, Z., Li, N., Zhang, H., Geng, N., A multi-objective dynamic scheduling approach using multiple attribute decision making in semiconductor manufacturing, International Journal of Production Economics, 130, (2011), 125-133.

[19] Nguyen, S., Zhang, M., Johnston, M., Tan, K., Automatic design of scheduling policies for dynamic multiobjective job shop scheduling via cooperative coevolution genetic programming, IEEE Transactions. Evolutionary Computation. 18, (2013), 193-208.

[20] Shen, X-N, Yao, X., Mathematical modeling and multi-objective evolutionary algorithms applied to dynamic flexible job shop scheduling problems, Information Sciences, 298, (2015), 198-224.

[21] Hoogeveen, H., Multicriteria scheduling, European Journal of Operational Research, 161, (2005), 592-623.

[22] Holthaus, O., Rajendran, C., Efficient dispatching rules for scheduling in a job shop, International Journal of Production Economics, 48, (1997), 87-105.

[23] Liu, J., MacCarthy, B. L., The classification of FMS scheduling problems, International Journal of Production Research, 34, (1996), 647-656.

[24] Muhlemann, A. P., Lockett, A. G., Farn, C. K., Job shop scheduling heuristics and frequency of scheduling, International Journal of Production Research, 20, (1982), 227-241.

[25] Law, A. M.; Kelton, W. D., Simulation Modeling and Analysis, Third Edition, McGraw-Hill, New-York, 2000.

[26] Jayamohan, M. S., Rajendran, C., New dispatching rules for shop scheduling: a step forward, International Journal of Production Research, 38, (2000), 563-586.

[27] Singh, A., Mehta, N. K., Jain, P. K., Performance analysis of flow time based dispatching rules with unreliable machines, International Journal of Simulation Modelling, 3, (2004), 109-120.

[28] Singh, A., Mehta, N. K., Jain, P. K., Tardiness based new dispatching rules for shop scheduling with unreliable machines, International Journal of Simulation Modelling, 4, (2005), 5-16. 